\title{
MicroECG: An Integrated Platform for the Cardiac Arrythmia Detection and Characterization
}

\author{
Bruno Nascimento ${ }^{1}$, Arnaldo Batista ${ }^{1}$, Luis Brandão Alves ${ }^{2}$, \\ Manuel Ortigueira ${ }^{1}$, and Raul Rato ${ }^{1}$ \\ ${ }^{1}$ Dept. of Electrical Engineering, Universidade Nova de Lisboa, Faculty of Sciences \\ and Technology, Quinta da Torre, 2825-516 Monte da Caparica, Portugal \\ brunonascimento@netcabo.pt, agb@fct.unl.pt, molafct.unl.pt, \\ rtr@fct.unl.pt \\ ${ }^{2}$ Department of Cardiology of Hospital Garcia de Orta, \\ Almada, Portugal \\ brandaoalves@gmail.com
}

\begin{abstract}
A software tool for the analysis of the High-Resolution Electrocardiogram (HR-ECG) for Arrhythmia detection is introduced. New algorithms based on Wavelet analysis are presented and compared with the classic Simson protocol over the $\mathrm{P}$ and QRS segments of the Electrocardiogram (EEG). A novel procedure based on a two step wavelet analysis and synthesis is performed in order to obtain a frequency description of the P, T or QRS segments. This frequency "signature" is useful for the detection of otherwise asymptomatic Arrhythmia patients. The tool has been developed in Matlab, and deployed for a standalone $\mathrm{C}$ application.
\end{abstract}

Keywords: Cardiac Arrhythmia, Fibrillation, Wavelets and Time-Frequency.

\section{Introduction}

Atrial fibrillation is the most common sustained heart arrhythmia. It is estimated to occur in more than 0.4 percent of the adult population and perhaps as many as 10 percent of the population who are 60 years or older [1]. Atrial arrhythmia may be transient or persistent. While most atrial arrhythmia occurs in individuals having other forms of underlying heart disease, some atrial arrhythmias occur independently. While atrial arrhythmias do not directly cause death as frequently as ventricular arrhythmias, they increase the risk factor for a number of other diseases such as strokes, thrombosis, atherosclerosis, systemic and cerebral embolism and cause a number of additional medical problems [2]. A two-step wavelet method is introduced for the detection and reconstruction of the cardiac micro-potentials [3] that account for the atrial and ventricular arrhythmia [4]. These algorithms are incorporated in a software platform tool (MicroECG) for the integrated High Resolution Electrocardiogram (HRECG) processing. A continuous wavelet method is herein applied for the detection of the micro potentials and a wavelet packet methodology for the reconstruction of these signals. MicroECG includes the following pre-processing tools: QRS detection, versatile peak detection, signal averaging or beat-by-beat analysis and ECG delineation 
with wavelet processes [5] [6]. These pre-processing tools are presented to the medical user or researcher in an intuitive and user-friendly interface where all the parameters can be viewed and changed. After this classical pre-processing is performed, an innovative wavelet methodology is applied in two steps. The first step consists in the application of the continuous wavelet transformation for the micro potentials detection via time-frequency scalograms. The next step includes the wavelet packet transform applied to the user selection in the scalogram plane in order to obtain both the reconstructed micro potentials and a frequency signature for each patient. This frequency signature contains information about the micro potentials presence in the patient's HR-ECG [7]. This presence is confirmed by higher frequency components. This procedure is user selected and is applied to the P-wave or the QRS complex. Fig. 1 shows a screen-shot of the MicroECG user interface presenting the Frank leads [8] automatically delineated ECG [9]. The system is versatile to the user and offers the pre-processing tools necessary to the wavelet processing [10].

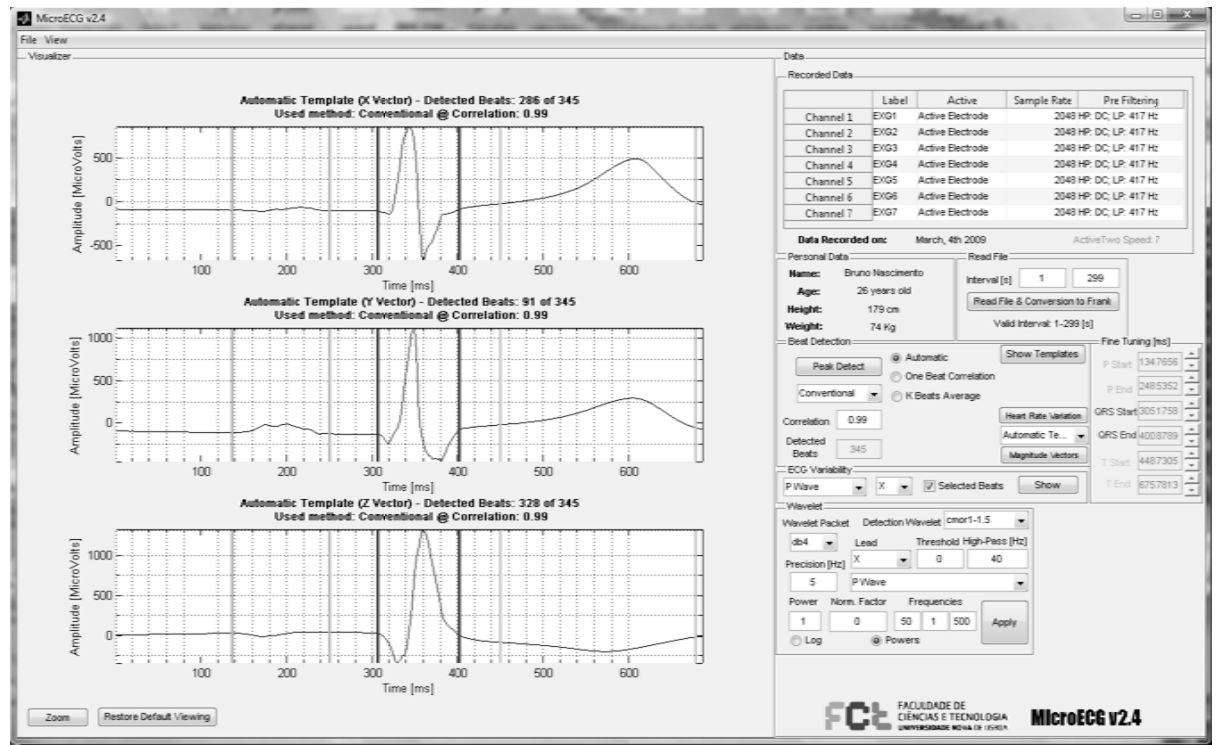

Fig. 1. A screen-shot of the MicroECG user interface showing the Frank leads automatically delineated ECG. The user can change a significant number of parameters.

\section{Contribution to Technological Innovation}

In this paper we present a tool with the following advanced features:

1. Incorporation under the same roof all the processing steps necessary to the high resolution HR-ECG processing thus saving the user all the time consuming pre - processing procedures.

2. User selection of all the HR-ECG analysis parameters. 
3. Innovative wavelet packet methodology for the detection and reconstruction of micro-potentials.

4. User driven (mouse-click) selection of signal features in the wavelet timescale plan for subsequent reconstruction.

5. Cardiac micro-potential syntheses and representation.

\section{Methods and Results}

\section{A. Continuous Wavelet transform}

The continuous wavelet transform (CWT) is based on a set of analyzing wavelets [11] that allow the decomposition of an electrocardiographic signal in a series of coefficients. Each wavelet used to analyze a signal has its own duration, temporal localization and frequency band. The resulting coefficients from the application of a continuous wavelet transformation corresponds to a measure of the components, on a given temporal and frequency band, of the HR-ECG segment under study. The resulting coefficients are obtained through two major steps:

1. The multiplication of an analyzing wavelet and the HR-ECG segment.

2. The calculation of the degree of correlation between the two signals above. This transformation is given by:

$$
C(a, b)=\frac{1}{\sqrt{a}} \int \operatorname{ECG}(t) \Psi\left(\frac{t-b}{a}\right) \cdot d t
$$

where $\mathbf{a}$ is the scale, $\mathbf{b}$ is the temporal segment, ECG (t) is the electrocardiogram and $\boldsymbol{\Psi}$ is the analyzing wavelet.

The P-wave scalogram shows an intense component below the $80 \mathrm{~Hz}$ mark and a weak component signal post this mark. Figure 2 shows the scalogram for a $40 \mathrm{~Hz}$ high-pass filtered simulated ECG signal with a micro-potential between 33 and 54.ms. [12] The rounded whiter patch represents micro-potential energy The colour intensity of the scalogram is proportional to the degree of correlation between the filtered signal and the detection wavelet at play. This means that the lighter zone in the scalogram represents the area where the signal is highly matched the detection wavelet [13]. It is known by the authors' experience that the detection wavelet cmor1-1.5 (Complex Morlet Wavelet) produces the best results regarding the detection of late potentials in the HR-ECG data, so it is set by default in the MicroECG software.

\section{B. Discrete wavelet transform}

Unlike the CWT, the Discrete Wavelet Transform (DWT) allows for signal reconstruction. Calculating wavelet coefficients at every possible scale produces a redundant signal representation. If the scales are based on powers of two - so-called dyadic grid - then the analysis will be much more efficient. This is known as the DWT. As seen in Fig. 3, the approximations $\left(\mathrm{cA}_{n}\right)$ are the high-scale, low-frequency components of the signal. The details $\left(\mathrm{cD}_{\mathrm{n}}\right)$ are the low-scale, high-frequency components. The decomposition process can be iterated, with successive approximations 

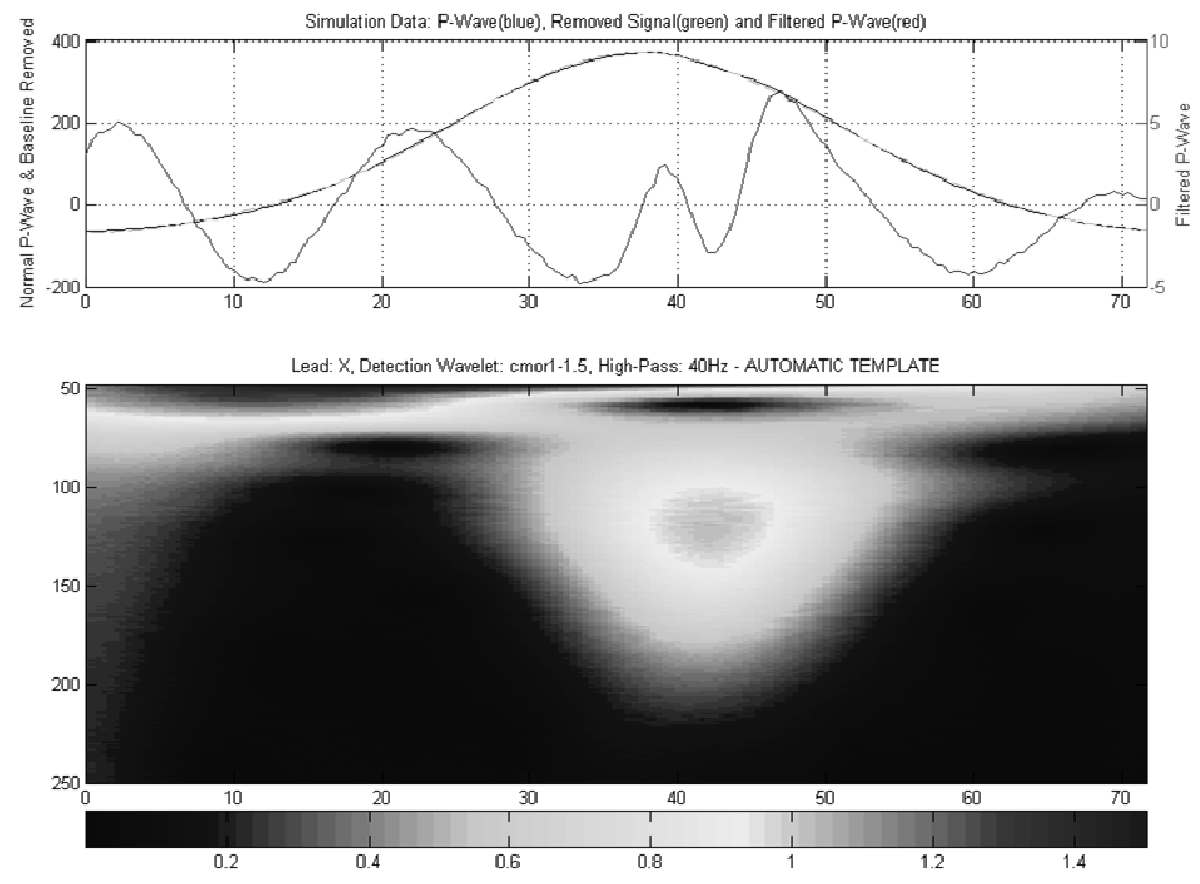

Fig. 2. Scalogram from a simulated signal showing a micro-potential event. Top plot: P wave with $380 \mu \mathrm{V}$ amplitude and the same wave after filtering with micro-potential around 33 and 54 $\mathrm{ms}$, and around $12 \mu \mathrm{V}$ amplitude.

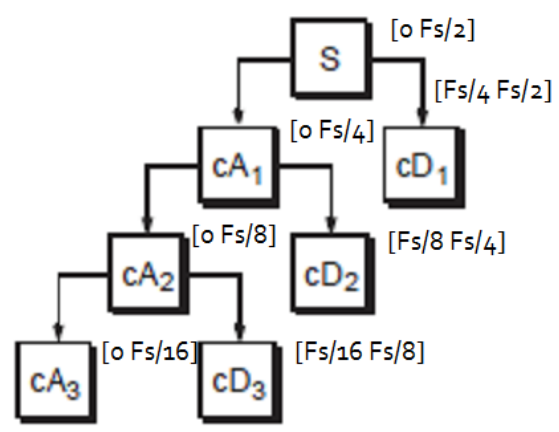

Fig. 3. Wavelet decomposition tree

being decomposed in turn, so that one signal is broken down into many lower resolution components. This is called the wavelet decomposition tree.

Since the analysis process is iterative, in theory it can be continued indefinitely. In reality, the decomposition can proceed only until the individual details consist of a single sample or pixel. 


\section{Wavelet packet transform}

The Discrete Wavelet Packet Transform (DWPT) offers a more complex and flexible analysis, because the details as well as the approximations are split.

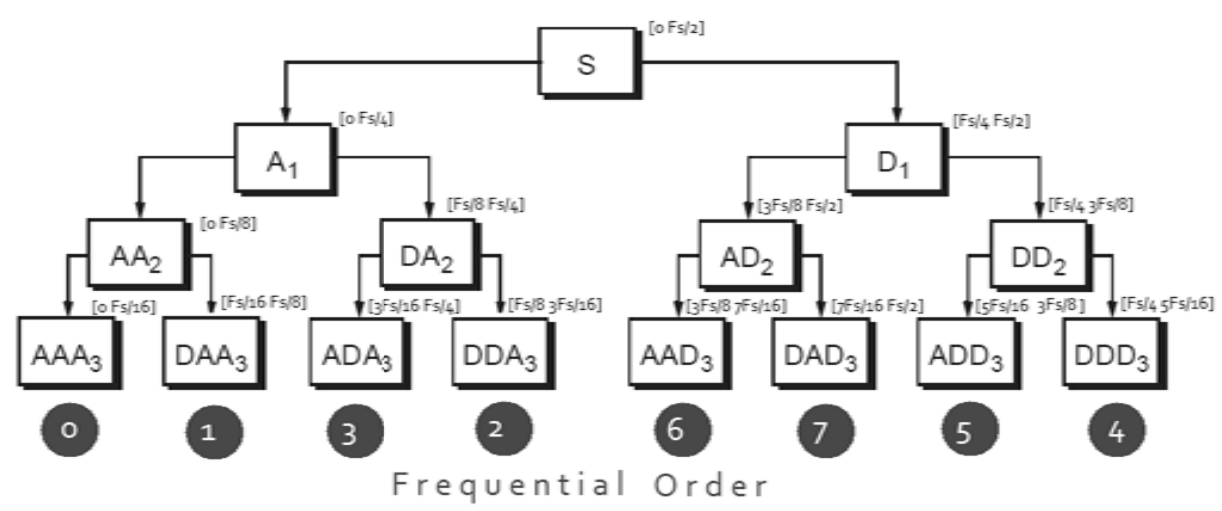

Fig. 4. Wavelet packet decomposition

The difference between DWT and DWPT is that the former presents the same frequency resolution in all the nodes in the same level, since the signal's approximation and detail nodes are all split into two new signals, instead of only the approximation node being split as it happens in the DWT as seen in Fig. 4. Therefore the DWPT turns out to be more efficient to study high resolution cases because there is no loss of resolution in the frequency axis. Another important thing to take notice is that in the final level of this multi-level decomposition "tree" there are the coefficients that can be used to reconstruct the original signal. However these coefficients or "leaves" of this "tree" are in no sequential order in terms of frequency, and one most take notice of this fact if signal reconstruction from this coefficients is required.

In MicroECG, these decomposition levels are calculated via the length of the signal and its sampling frequency. The number of "leaves" of this wavelet packet decomposition "tree" is always a power of 2, for example, if a number of 8 levels are calculated there will be 256 "leaves" or coefficients in the end. However, all these 256 "leaves" will be shown only if the user selects all frequencies to be analyzed from zero to the Nyquist frequency. If the user only selects a sub-set of the frequencies available to be analyzed the 256 "leaves" will be reduced accordingly. For better exemplify these same principles, Fig. 5 is shown where the micro-potential lies around 33-44ms with less than $10 \mu \mathrm{V}$ amplitude and bandwidth between 65 and $187 \mathrm{~Hz}$.

Figure 6 shows the colour maps DPWT energy map of: Top: a patient with Paroxistic Atrial Fibrillation; Bottom: a normal subject. Lighter colour means increased node energy, therefore indicating micro-potential activity. 

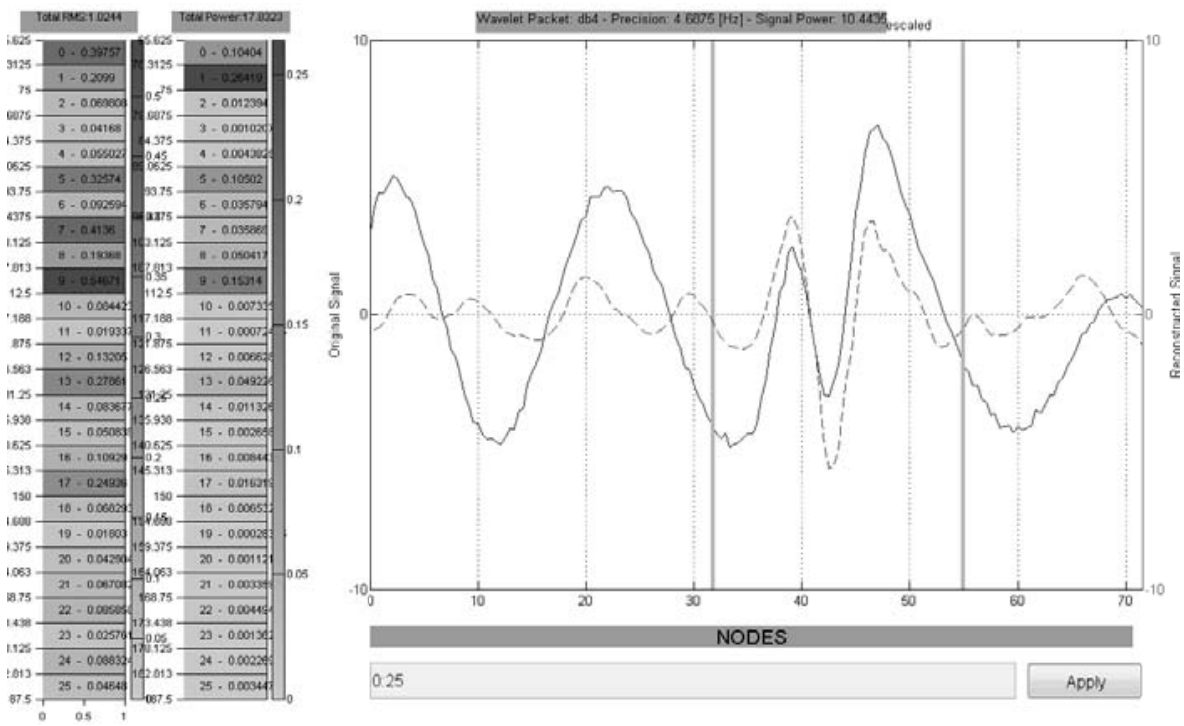

Fig. 5. DPWT reconstruction for the micro-potential of Fig. 2 (dashed) and original filtered $P$ wave. The micro-potential lies around $33-44 \mathrm{~ms}$ with less than $10 \mu \mathrm{V}$ amplitude and bandwidth between 65 and $187 \mathrm{~Hz}$.

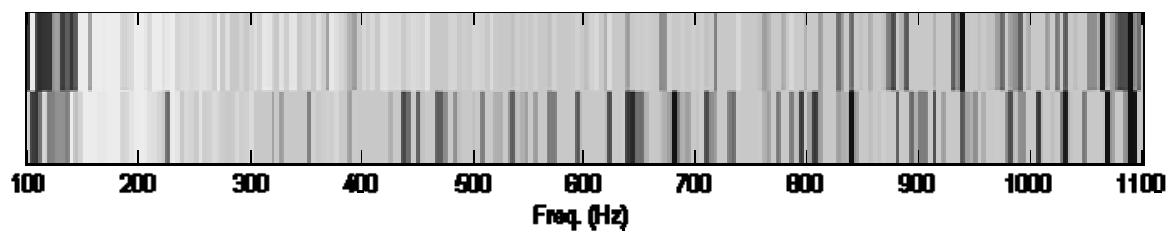

Fig. 6. DPWT energy map of: Top: a patient with Paroxistic Atrial Fibrillation; Bottom: a normal subject

\section{Conclusions and Future Work}

The DWPT is a promising tool in the detection of small amplitude, short lived signals present in the HR-ECG. A spectral signature is obtained and the reconstruction of the micro-potentials is possible through the selection of the most energetic nodes. In this work, interesting results have been obtained with simulated data, and will now progress with real data from patients with Paroxistic Atrial Fibrillation. The MicroECG system will serve as a platform for this research work relieving the user from the time consuming pre-processing steps along with all the wavelet algorithms. This platform is design to be flexible and open to improvements. 


\section{References}

1. Cox, J.L., et al.: Electrophysiology, Pacing and Arrhythmia, Operations for Atrial Fibrillation. Clin. Cardiol. 14, 827-834 (1991)

2. Chang, Y.U., et al.: National Implications for Rhythm Management and Stroke Prevention: the AnTicoagulation and Risk Factors. Atrial Fibrillation (ATRIA) Study, JAMA 285, 2370-2375 (2001)

3. Kuchar, D., Thorburn, C., Sammel, F.: Late potentials detected after myocardial infarction. Natural history and prognostic significance. Circulation 6, 1280-1289 (1986)

4. Zandi, A.S., Moradi, M.H.: Quantitative evaluation of a wavelet-based method in ventricular late potential detection. The Journal of Pattern Recognition Society (2006)

5. Daubechies, I.: Ten Lectures on Wavelets. Society for Industrial and Applied Mathematics (1992)

6. Malmivuo, J., Plonsey, R.: Bioelectromagnetism - Principles and Applications of Bioelectric and Biomagnetic Fields. Oxford University Press, New York (1995)

7. Berbari, E.J., Steinberg, J.S.: A practical guide to the use of the high-resolution electrocardiography. Wiley-Blackwell (2000)

8. Frank, E.: An accurate, clinically practical system for spatial vectorcardiography. Circulation 13, 737-749 (1956)

9. Almeida, R., Martínez, J.P.: Multilead ECG Delineation Using Spatially Projected Leads From Wavelet Transform Loops. IEEE Transactions on Biomedical Engineering 56(8) (2009)

10. Tompkins, W.J.: Biomedical Digital Signal Processing: C Language Examples and Laboratory Experiments for the IBM PC. Prentice Hall, Englewood Cliffs (1993)

11. Couderc, J.P., Zareba, W.: Contribution of wavelets to the non-invasive Electrocardiology. In: ANE, pp. 54-62 (1993)

12. Afonso, V., Tompkins, W., Nguyen, T., Luo, S.: ECG beat detection using filter banks. Trans. Biomed. Eng. 46(2), 192-202 (1999)

13. Natwong, B., Sooraksa, P.: Wavelet Entropy Analysis of the High Resolution ECG, Industrial Electronics and Applications. In: 1ST IEEE Conference (2006) 